\title{
Some Associated Curves of Normal Indicatrix of a Regular Curve
}

\author{
Nidal ECHABBI ${ }^{1}$, Amina OUAZZANI CHAHDI $^{2}$ \\ ${ }^{1}$ Department of Mathematics, Ben M'Sik Faculty of Science, Hassan II Mohammadia-Casablanca University, Morocco \\ Correspondence: Nidal ECHABBI, Department of Mathematics, Ben M'Sik Faculty of Science, Hassan II Mohammadia- \\ Casablanca University, Morocco
}

Received: November 17, 2019 Accepted: December 23, 2019 Online Published: January 31, 2020

doi:10.5539/jmr.v12n1p84

URL: https://doi.org/10.5539/jmr.v12n1p84

\begin{abstract}
In this paper, we consider integral curves of a vector field generated by Frenet vectors of normal indicatrix of a given curve in Euclidean 3-space. We define some new associated curves such as evolute direction curves, Bertrand direction curves and Mannheim directon curves of the normal indicatrix of a regular curve, respectively. We also found the relationships between curvatures of these curves. By using these associated curves, we give a new approach to construct slant helices and C- slant helices. Finally, we present some examples.
\end{abstract}

Keywords: associated curves, direction curves, evolute curve, Bertrand curve, Mannheim curve, slant helix, $C$ - slant helix

\section{Introduction}

In differential geometry, the curves that found in a differential and mathematical relationship between two or more curves are called associated curves. Among these curves the most studied ones are evolute curve, Bertrand curve, Mannheim curve, and spherical indicatrix...

Recently, a new type of associative curves called direction curves have been introduced by J. H. Choi \& Y. H. Kim (2012) as the integral curves of some vector fields generated by the Frenet frame along the curve. Then, they found some relations between curvatures of these associated curves. Moreover, by using these curves, they gave a canonical method to construct general helices and slant helices.

This new type of associative curves has attracted the attention of many differential geometers. In (J. H. Choi, Y. H. Kim \& A. T. Ali (2012)) \& (J. Qian \& Y. H. Kim (2015)), a direction curve was studied in Minkowski space $E_{1}^{3}$, in T. Kőrpinar, M. T. Sariaydin \& E. Turhan (2013) this curve was studied by using Bishop frame instead of Frenet frame. In N. Macit \& M. Düldül (2014) the authors have used the unit Darboux vector field $W$ of a given curve to define the $W$-direction curve. They also introduced $V$-direction curve of a given curve on a surface by using Darboux frame, and examined direction curve of a given curve in four-dimensional Euclidean space. Moreover, M. Önder (2017) has studied curve couples with consideration of direction curve and donor curve, also some new associated curves are defined by using direction curves of tangent indicatrix of a given curve in B. Şahiner (2019).

In this paper, we define $X$-direction curve as the integral curve of unit vector field taken from the Frenet frame along the normal indicatrix of a given regular curve. By utilizing of this definition, we define some new associative curves, such as evolute-direction curve, Bertrand-direction curve and Mannheim-direction curve of the normal indicatrix of a given curve, respectively. We determine some relationships between curvatures of these curves. Through this study, a new approach to construct slant helices and C-slant helices is given. Finally, we present some illustrated examples.

\section{Preliminaries}

In this section we recall some basic concepts and properties on classical differential geometry of space curves in the Euclidean 3-space.

- We denote by $E^{3}$ the Euclidean 3-space, with the usual metric

$$
\langle x, y\rangle=x_{1} y_{1}+x_{2} y_{2}+x_{3} y_{3},
$$

where $x=\left(x_{1}, x_{2}, x_{3}\right)$ and $y=\left(y_{1}, y_{2}, y_{3}\right)$ are two vectors of $E^{3}$.

- Let $\alpha=\alpha(s): \quad I \subset \mathbb{R} \rightarrow E^{3}$ be a regular curve in $E^{3}$, with non null curvature, we also assume that is parametrized by arc-length $s$, i.e, $\left\langle\alpha^{\prime}(s), \alpha^{\prime}(s)\right\rangle=1$ for all $s \in I$. 
- In each point of a space curve we have a moving frame, in particular the Frenet frame and the alternative frame.

The Frenet frame along the curve $\alpha$, is an orthonormal frame $(T(s), N(s), B(s))$,

$$
T(s)=\frac{d \alpha(s)}{d s}, \quad N(s)=\frac{1}{k(s)} T^{\prime}(s), B(s)=T(s) \times N(s),
$$

where $T$ is the unit tangent, $N$ is the unit principal normal, $B$ is the unit binormal and $k$ et $\tau$ are respectively the curvature and the torsion of the curve $\alpha$, given by

$$
k(s)=\left\|\alpha^{\prime \prime}(s)\right\| \quad \text { et } \quad \tau(s)=-\left\langle B^{\prime}(s), N(s)\right\rangle .
$$

Then, the Frenet equations are given by the following relations:

$$
\frac{d}{d s}\left[\begin{array}{c}
T(s) \\
N(s) \\
B(s)
\end{array}\right]=\left[\begin{array}{ccc}
0 & k(s) & 0 \\
-k(s) & 0 & \tau(s) \\
0 & -\tau(s) & 0
\end{array}\right]\left[\begin{array}{c}
T(s) \\
N(s) \\
B(s)
\end{array}\right] .
$$

The authors U. Beyhan, İ. Gök, \& Y. Yaylı. (2016), have introduced the alternative frame $(N, C, W)$, where

$$
N, C=\frac{N^{\prime}}{\left\|N^{\prime}(s)\right\|} \text { et } W=\frac{\tau T+k B}{\sqrt{\tau^{2 \cdot}+k^{2}}},
$$

are respectively the unit principal normal, the derivative of the principal normal and the unit Darboux vector field along the curve $\alpha$. Then, the derivative formulas of the alternative frame are given by the following relations:

$$
\frac{d}{d s}\left[\begin{array}{c}
N(s) \\
C(s) \\
W(s)
\end{array}\right]=\left[\begin{array}{ccc}
0 & f(s) & 0 \\
-f(s) & 0 & g(s) \\
0 & -g(s) & 0
\end{array}\right]\left[\begin{array}{c}
N(s) \\
C(s) \\
W(s)
\end{array}\right],
$$

where $f(s)=\sqrt{\tau^{2}+k^{2}}$ and $g(s)=\frac{k^{2}}{\tau^{2}+k^{2}}\left(\frac{\tau}{k}\right)^{\prime}$ are differentiable functions.

- The curve $\alpha$ whose tangent vector $T(s)$ makes a constant angle with a fixed direction is called general helix. $\alpha$ is a general helix if and only if the following function

$$
h(s)=\frac{\tau}{k}(s)
$$

is constant.

- The curve $\alpha$ whose principal normal vector $N(s)$ makes a constant angle with a fixed direction is called slant helix. $\alpha$ is a slant helix if and only if the following function

$$
\sigma(s)=\frac{k^{2}}{\left(k^{2}+\tau^{2}\right)^{\frac{3}{2}}}\left(\frac{\tau}{k}\right)^{\prime}(s)
$$

is constant.

- The curve $\alpha$ whose vector field $C(s)$ makes a constant angle with a fixed direction is called C-slant helix. $\alpha$ is a C-slant helix if and only if the following function

$$
\Gamma(s)=\frac{f^{2}}{\left(f^{2}+g^{2}\right)^{\frac{3}{2}}}\left(\frac{g}{f}\right)^{\prime}(s)=\frac{\sigma^{\prime}}{f\left(1+\sigma^{2}\right)^{\frac{3}{2}}}(s)
$$

is constant.

- Let $\alpha: I \subset \mathbb{R} \rightarrow E^{3}$ and $\beta: I \subset \mathbb{R} \rightarrow E^{3}$ be two curves in $E^{3}$, with Frenet frame $\left\{T_{\alpha}, N_{\alpha}, B_{\alpha}\right\}$ and $\left\{T_{\beta}, N_{\beta}, B_{\beta}\right\}$ respectively. $\beta$ is called evolute (resp. Bertrand, Mannheim) curve of $\alpha$ if and only if $N_{\beta}=T_{\alpha}$ (resp. $\left.N_{\beta}=N_{\alpha}, N_{\beta}=B_{\alpha}\right)$.

- Let $\quad \alpha: I \subset \mathbb{R} \rightarrow E^{3}$ be a regular curve in $E^{3}$. If we translate of the principal normal vector field to the center of unit sphere $S^{2}$ in the $E^{3}$, we obtain a spherical curve $\alpha_{N}\left(s_{N}\right)=N(s)$. This curve is called normal indicatrix of curve $\alpha=\alpha(s)$. 
Denote by $\left\{T_{N}, N_{N}, B_{N}\right\}$ the Frenet frame of $\alpha_{N}$ and by $k_{N}, \tau_{N}$ its curvatures. Then, the Frenet equations are given by the following relations:

$$
\frac{d}{d s_{N}}\left[\begin{array}{c}
T_{N}\left(s_{N}\right) \\
N_{N}\left(s_{N}\right) \\
B_{N}\left(s_{N}\right)
\end{array}\right]=\left[\begin{array}{ccc}
0 & k_{N}\left(s_{N}\right) & 0 \\
-k_{N}\left(s_{N}\right) & 0 & \tau_{N}\left(s_{N}\right) \\
0 & -\tau_{N}\left(s_{N}\right) & 0
\end{array}\right]\left[\begin{array}{c}
T_{N}\left(s_{N}\right) \\
N_{N}\left(s_{N}\right) \\
B_{N}\left(s_{N}\right)
\end{array}\right]
$$

where

$$
\left\{\begin{array}{l}
T_{N}=\frac{-T+h B}{\sqrt{1+h^{2}}} \\
N_{N}=\frac{\sigma}{\sqrt{1+\sigma^{2}}}\left[\frac{h T+B}{\sqrt{1+h^{2}}}-\frac{N}{\sigma}\right], \\
B_{N}=\frac{1}{\sqrt{1+\sigma^{2}}}\left[\frac{h T+B}{\sqrt{1+h^{2}}}+\sigma N\right],
\end{array}\right.
$$

and

$$
k_{N}=\sqrt{1+\sigma^{2}}, \tau_{N}=\Gamma \sqrt{1+\sigma^{2}},
$$

with

$$
\Gamma=\frac{\sigma^{\prime}}{k \sqrt{1+h^{2}}\left(1+\sigma^{2}\right)^{\frac{3}{2}}},
$$

and $s_{N}$ is the arc-length parameter of $\alpha_{N}$.

\section{Direction Curves of Normal Indicatrix}

Let $\alpha=\alpha(s)$ be a regular curve in $E^{3}$ with the Frenet frame $\{T, N, B\}$ and $\alpha_{N}=\alpha_{N}\left(s_{N}\right)$ its normal indicatrix with the Frenet frame $\left\{T_{N}, N_{N}, B_{N}\right\}$. For the normal indicatrix $\alpha_{N}$, consider a vector field $X$ given by

$$
X\left(s_{N}\right)=x\left(s_{N}\right) T_{N}\left(s_{N}\right)+y\left(s_{N}\right) N_{N}\left(s_{N}\right)+z\left(s_{N}\right) B_{N}\left(s_{N}\right),
$$

where $x, y$ and $z$ are real functions of $s_{N}$ the arc-length parameter of the normal indicatrix of a curve $\alpha$.

As a particular case, we limit our study to a unit vector field $X$, then we get:

$$
x^{2}\left(s_{N}\right)+y^{2}\left(s_{N}\right)+z^{2}\left(s_{N}\right)=1 .
$$

By differentiating equation (3), we have the following:

$$
x\left(s_{N}\right) x^{\prime}\left(s_{N}\right)+y\left(s_{N}\right) y^{\prime}\left(s_{N}\right)+z\left(s_{N}\right) z^{\prime}\left(s_{N}\right)=0 .
$$

Then the definition of $X$-direction curve of the normal indicatrix $\alpha_{N}$ of a curve $\alpha$ is given as follows:

Definition 1. Let $\alpha$ be a curve in $E^{3}, \alpha_{N}$ be the normal indicatrix of a curve $\alpha$ and $X$ be the unit vector field satisfies (2) and (3). The integral curve of $X$ is called $X$-direction curve of $\alpha_{N}$.

Let $\beta=\beta\left(s_{\beta}\right)$ be a $X$-direction curve of the normal indicatrix $\alpha_{N}$ of a curve $\alpha$, with the Frenet frame $\left\{T_{\beta}, N_{\beta}, B_{\beta}\right\}$ and the curvatures $\left\{k_{\beta}, \tau_{\beta}\right\}$. Since

$$
\frac{d \beta}{d s_{N}}=X\left(s_{N}\right)
$$

we have

$$
\frac{d \beta}{d s_{\beta}} \frac{d s_{\beta}}{d s_{N}}=X\left(s_{N}\right)
$$

where $s_{\beta}$ is the arc-length parameter of $\beta$.

By passing to the norm we obtain: $s_{\beta}=s_{N}+c$. Without loss of generality, we assume that $s_{\beta}=s_{N}$.

By differentiating (2), we have the following:

$$
k_{\beta} N_{\beta}=\left(x \prime-y k_{N}\right) T_{N}+\left(y^{\prime}+x k_{N}-z \tau_{N}\right) N_{N+}\left(z^{\prime}+y \tau_{N}\right) B_{N} .
$$

By using the equation (5), we construct a new directon curves such as evolute-direction curves, Bertrand-direction curves and Mannheim-directon curves of the normal indicatrix $\alpha_{N}$ of $\alpha$, and we give some properties characterizing these curves. 


\subsection{Evolute-Direction Curves of Normal Indicatrix}

In this section, we characterize the $X$-direction curves of the normal indicatrix $\alpha_{N}$ of a regular curve $\alpha$ which are evolute of $\alpha_{N}$. As a result, we obtain some relations between these curves.

Theorem 2. Let $\alpha$ be a curve in $E^{3}$ and $\alpha_{N}$ be normal indicatrix of a curve $\alpha$.

An $X$-direction curve of $\alpha_{N}$ is its evolute if and only if

$$
X=\sin \left(\int \tau_{N}\left(s_{N}\right) d s_{N}\right) N_{N}+\cos \left(\int \tau_{N}\left(s_{N}\right) d s_{N}\right) B_{N} .
$$

Proof. Let $\beta$ be $X$-direction curve of $\alpha_{N}$, and its evolute. By using (5) we obtain the following system of differential equations :

$$
\left\{\begin{array}{ccc}
x^{\prime}-y k_{N} & =k_{\beta}, \\
y^{\prime}+x k_{N}-z \tau_{N} & =0 \\
z^{\prime}+y \tau_{N} & =0
\end{array}\right.
$$

By multiplying the first, the second and the third equation in (6) with $x, y$ and $z$ respectively and by adding the results we obtain, $x=0$. Since $y^{2}+z^{2}=1$, we have:

$$
y=\sin \left(\theta\left(s_{N}\right)\right), z=\cos \left(\theta\left(s_{N}\right)\right) .
$$

By giving $x$ the value 0 in (6) and using (7), we obtain:

$$
\theta\left(s_{N}\right)=\int \tau_{N}\left(s_{N}\right) d s_{N}
$$

Hence the vector coordinates of $X\left(s_{N}\right)$ are given by

$$
\left\{x\left(s_{N}\right)=0, \quad y\left(s_{N}\right)=\sin \left(\int \tau_{N}\left(s_{N}\right) d s_{N}\right), \quad z\left(s_{N}\right)=\cos \left(\int \tau_{N}\left(s_{N}\right) d s_{N}\right)\right\} .
$$

Conversely, let $X=\sin \left(\int \tau_{N} d s_{N}\right) N_{N}+\cos \left(\int \tau_{N} d s_{N}\right) B_{N}$, we easily prove that $N_{\beta}=\frac{X^{\prime}}{\left\|X^{\prime}\right\|}=T_{N}$.

From Theorem (2), we can give the following definition:

Definition 3. An integral curve of vector field $\sin \left(\int \tau_{N} d s_{N}\right) N_{N}+\cos \left(\int \tau_{N} d s_{N}\right) B_{N}$ is called evolute-direction curve of $\alpha_{N}$ the normal indicatrix of a curve $\alpha$.

In the following, we give the relationships between curvatures of the normal indicatrix $\alpha_{N}$ of a regular curve $\alpha$ and its evolute-direction curve $\beta$.

Proposition 4. Let $\alpha$ be a curve in $E^{3}, \alpha_{N}$ be the normal indicatrix of a curve $\alpha$ and $\beta$ is evolute-direction curve of $\alpha_{N}$. The relationships between curvatures of the curves $\alpha_{N}$ and $\beta$ can be expressed as follows:

$$
k_{\beta}=-k_{N} \sin \left(\int \tau_{N}\left(s_{N}\right) d s_{N}\right), \quad \tau_{\beta}=k_{N} \cos \left(\int \tau_{N}\left(s_{N}\right) d s_{N}\right)
$$

and

$$
k_{N}=\sqrt{k_{\beta}^{2}+\tau_{\beta}^{2}}, \quad \tau_{N}=\frac{k_{\beta}^{2}}{k_{\beta}^{2}+\tau_{\beta}^{2}}\left(\frac{\tau_{\beta}}{k_{\beta}}\right)^{\prime} .
$$

Proof. From (6), it follows

$$
k_{\beta}=-k_{N} \sin \left(\int \tau_{N}\left(s_{N}\right) d s_{N}\right)
$$

Since $\beta$ is an evolute-direction curve of $\alpha_{N}$, then the Frenet frame of a curve $\beta$ can be expressed by:

$$
\left\{\begin{array}{l}
T_{\beta}=\sin \left(\int \tau_{N}\left(s_{N}\right) d s_{N}\right) N_{N}+\cos \left(\int \tau_{N}\left(s_{N}\right) d s_{N}\right) B_{N} \\
N_{\beta}=T_{N} \\
B_{\beta}=\cos \left(\int \tau_{N}\left(s_{N}\right) d s_{N}\right) N_{N}-\sin \left(\int \tau_{N}\left(s_{N}\right) d s_{N}\right) B_{N}
\end{array}\right.
$$


By the definition, we obtain the torsion as following:

$$
\tau_{\beta}=k_{N} \cos \left(\int \tau_{N}\left(s_{N}\right) d s_{N}\right) .
$$

From (8) and (9), we have:

$$
k_{N}=\sqrt{k_{\beta}^{2}+\tau_{\beta}^{2}},
$$

and

$$
\int \tau_{N}\left(s_{N}\right) d s_{N}=-\operatorname{arccot}\left(\frac{\tau_{\beta}}{k_{\beta}}\right) .
$$

By differentiating the last equality, we obtain:

$$
\tau_{N}=\frac{k_{\beta}^{2}}{k_{\beta}^{2}+\tau_{\beta}^{2}}\left(\frac{\tau_{\beta}}{k_{\beta}}\right)^{\prime} .
$$

Corollary 5. Let $\beta$ be evolute-direction curve of $\alpha_{N}$, normal indicatrix of a curve $\alpha$. Then:

$$
\Gamma=\frac{\tau_{N}}{k_{N}}=\frac{k_{\beta}^{2}}{\left(k_{\beta}^{2}+\tau_{\beta}^{2}\right)^{\frac{3}{2}}}\left(\frac{\tau_{\beta}}{k_{\beta}}\right)^{\prime} .
$$

By using (1) and from corollary (5), we can give the two following theorems without any proof.

Theorem 6. Let $\alpha$ be a curve in $E^{3}, \alpha_{N}$ be normal indicatrix of a curve $\alpha$ and $\beta$ be evolute-direction curve of $\alpha_{N}$. Then the following are equivalents:

i- $\alpha$ is a slant helix.

ii- $\alpha_{N}$ is a circle or a part of a circle on unit sphere.

iii- $\beta$ is a general helix.

Theorem 7. Let $\alpha$ be a curve in $E^{3}, \alpha_{N}$ be normal indicatrix of a curve $\alpha$ and $\beta$ be evolute-direction curve of $\alpha_{N}$. Then the following are equivalents:

i- $\alpha$ is a C-slant helix.

ii- $\alpha_{N}$ is a spherical helix.

iii- $\beta$ is a slant helix.

\subsection{Bertrand-Direction Curves of Normal Indicatrix}

Like the evolute-direction curves, in this section, we give a characterization of the $X$-direction curves of $\alpha_{N}$ which are Bertrand of $\alpha_{N}$. As a result, some relations between these curves are determined.

Theorem 8. Let $\alpha$ be a curve in $E^{3}$ and $\alpha_{N}$ be normal indicatrix of a curve $\alpha$.

An X-direction curve of $\alpha_{N}$ is its Bertrand if and only if

$$
X=\cos \theta T_{N}+\sin \theta B_{N}
$$

where $\theta$ is a constant angle.

Proof. Let $\beta$ be X-direction curve of $\alpha_{N}$ and Bertrand of $\alpha_{N}$. By using (5) we obtain the following system of differential equations:

$$
\left\{\begin{array}{ccc}
x^{\prime}-y k_{N} & = & 0, \\
y^{\prime}+x k_{N}-z \tau_{N} & = & k_{\beta}, \\
z^{\prime}+y \tau_{N} & = & 0 .
\end{array}\right.
$$


By multiplying the first, the second and the third equations in (10) with $x, y$ and $z$ respectively and by adding the results we obtain $y=0$. By giving $y$ the value 0 in (10), we obtain:

$$
x=c_{1}, z=c_{2},
$$

where $c_{1}$ and $c_{2}$ are constants real numbers. Since $x^{2}+z^{2}=1$, we have the vector coordinates of $X\left(s_{N}\right)$

$$
\left\{x\left(s_{N}\right)=\cos (\theta), \quad y\left(s_{N}\right)=0, \quad z\left(s_{N}\right)=\sin (\theta)\right\} .
$$

where $\theta$ is a constant angle between the tangent vector of the curve $\alpha_{N}$ and the tangent vector of the curve $\beta$.

Conversely, let $X=\cos (\theta) T_{N}+\sin (\theta) B_{N}$, we easily prove that $N_{\beta}=\frac{X^{\prime}}{\left\|X^{\prime}\right\|}=N_{N}$.

From Theorem (8), we can give the following definition:

Definition 9. An integral curve of vector field $\cos (\theta) T_{N}+\sin (\theta) B_{N}$ is called Bertrand-direction curve of $\alpha_{N}$ the normal indicatrix of a curve $\alpha$.

In the following, we give the relationships between curvatures of the normal indicatrix of a regular curve and its Bertranddirection curves.

Proposition 10. Let $\alpha$ be a curve in $E^{3}, \alpha_{N}$ be normal indicatrix of a curve $\alpha$ and $\beta$ is Bertrand-direction curve of $\alpha_{N}$. The relationships between curvatures of the curves $\alpha_{N}$ and $\beta$ can be expressed as follows:

$$
k_{\beta}=k_{N} \cos (\theta)-\tau_{N} \sin (\theta), \quad \tau_{\beta}=k_{N} \sin (\theta)+\tau_{N} \cos (\theta) .
$$

Proof. From (10) we have

$$
k_{\beta}=k_{N} \cos (\theta)-\tau_{N} \sin (\theta),
$$

Since $\beta$ is a Bertrand-direction curve of $\alpha_{N}$, then the Frenet frame of a curve $\beta$ can be expressed by:

$$
\left\{\begin{array}{l}
T_{\beta}=\cos (\theta) T_{N}+\sin (\theta) B_{N} \\
N_{\beta}=N_{N} \\
B_{\beta}=-\sin (\theta) T_{N}+\cos (\theta) B_{N} .
\end{array}\right.
$$

By the definition, we obtain the torsion as following:

$$
\tau_{\beta}=k_{N} \sin (\theta)+\tau_{N} \cos (\theta) .
$$

Corollary 11. Let $\beta$ be Bertrand-direction curve of $\alpha_{N}$, the normal indicatrix of a curve $\alpha$, then

$$
\begin{aligned}
\Gamma & =\frac{-\sin (\theta)+\frac{\tau_{\beta}}{k_{\beta}} \cos (\theta)}{\cos (\theta)+\frac{\tau_{\beta}}{k_{\beta}} \sin (\theta)}, \\
\frac{k_{N}^{2}}{\left(k_{N}^{2}+\tau_{N}^{2}\right)^{\frac{3}{2}}}\left(\frac{\tau_{N}}{k_{N}}\right)^{\prime} & =\frac{k_{\beta}^{2}}{\left(k_{\beta}^{2}+\tau_{\beta}^{2}\right)^{\frac{3}{2}}}\left(\frac{\tau_{\beta}}{k_{\beta}}\right)^{\prime} .
\end{aligned}
$$

Proof. From the proposition (10), it can be easily seen that:

$$
k_{N}=k_{\beta} \cos (\theta)+\tau_{\beta} \sin (\theta), \quad \tau_{N}=-k_{\beta} \sin (\theta)+\tau_{\beta} \cos (\theta) .
$$

We have

$$
\frac{\tau_{N}}{k_{N}}=\frac{-\sin (\theta)+\frac{\tau_{\beta}}{k_{\beta}} \cos (\theta)}{\cos (\theta)+\frac{\tau_{\beta}}{k_{\beta}} \sin (\theta)},
$$

by differentiating the last report, we obtain

$$
\left(\frac{\tau_{N}}{k_{N}}\right)^{\prime}=\frac{1}{\left(\cos (\theta)+\frac{\tau_{\beta}}{k_{\beta}} \sin (\theta)\right)^{2}}\left(\frac{\tau_{\beta}}{k_{\beta}}\right)^{\prime} .
$$


Since

$$
k_{N}^{2}+\tau_{N}^{2}=k_{\beta}^{2}+\tau_{\beta}^{2} \quad \text { and } \quad k_{N}^{2}=\left(\cos (\theta)+\frac{\tau_{\beta}}{k_{\beta}} \sin (\theta)\right)^{2} k_{\beta}^{2},
$$

therefore we complete the proof.

From corollary (11), we have the two following theorems without any proof.

Theorem 12. Let $\alpha$ be a curve in $E^{3}, \alpha_{N}$ be normal indicatrix of a curve $\alpha$ and $\beta$ be Bertrand-direction curve of $\alpha_{N}$. Then $\alpha$ is a $C$-slant helix if and only if $\beta$ is a general helix.

Theorem 13. Let $\alpha$ be a curve in $E^{3}, \alpha_{N}$ be normal indicatrix of a curve $\alpha$ and $\beta$ be Bertrand-direction curve of $\alpha_{N}$. Then:

$\alpha_{N}$ is a spherical helix if and only if $\beta$ is a general helix.

$\alpha_{N}$ is a spherical slant helix if and only if $\beta$ is a slant helix.

\subsection{Mannheim-Direction Curves of Normal Indicatrix}

In this section, we give a characterization of $X$-direction curves of $\alpha_{N}$ which are Mannheim of $\alpha_{N}$ and obtain some relations between these curves.

Theorem 14. Let $\alpha$ be a curve in $E^{3}$ and $\alpha_{N}$ be normal indicatrix of a curve $\alpha$.

An X-direction curve of $\alpha_{N}$ is its Mannheim if and only if

$$
X=\sin \left(\int k_{N}\left(s_{N}\right) d s_{N}\right) T_{N}+\cos \left(\int k_{N}\left(s_{N}\right) d s_{N}\right) N_{N} .
$$

Proof. We suppose that $\beta$ is $X$-direction curve of $\alpha_{N}$ and its Mannheim. By using (5) we obtain the following system of differential equations:

$$
\left\{\begin{array}{ccc}
x^{\prime}-y k_{N} & = & 0, \\
y^{\prime}+x k_{N}-z \tau_{N} & = & 0, \\
z^{\prime}+y \tau_{N} & = & k_{\beta} .
\end{array}\right.
$$

By multiplying the first, the second and the third equations in (11) with $x, y$ and $z$ respectively and by adding the results we obtain, $z=0$. Since $x^{2}+y^{2}=1$, then:

$$
x=\sin \left(\theta\left(s_{N}\right)\right), y=\cos \left(\theta\left(s_{N}\right)\right) .
$$

By giving $\mathrm{z}$ the value 0 in (11) and using (12), we obtain:

$$
\theta\left(s_{N}\right)=\int k_{N}\left(s_{N}\right) d s_{N} .
$$

Hence the vector coordinates of $X\left(s_{N}\right)$ are given by

$$
\left\{x\left(s_{N}\right)=\sin \left(\int k_{N}\left(s_{N}\right) d s_{N}\right), \quad y\left(s_{N}\right)=\cos \left(\int k_{N}\left(s_{N}\right) d s_{N}\right), \quad z\left(s_{N}\right)=0\right\} .
$$

Conversely, let $X=\sin \left(\int k_{N}\left(s_{N}\right) d s_{N}\right) T_{N}+\cos \left(\int k_{N}\left(s_{N}\right) d s_{N}\right) N_{N}$, we easily prove that $N_{\beta}=\frac{X^{\prime}}{\left\|X^{\prime}\right\|}=B_{N}$.

From Theorem (14), we can give the following definition:

Definition 15. An integral curve of vector field $\sin \left(\int k_{N} d s_{N}\right) T_{N}+\cos \left(\int k_{N} d s_{N}\right) N_{N}$ is called Mannheim-direction curve of $\alpha_{N}$ the normal indicatrix of a curve $\alpha$.

In the following, we give the relationships between curvatures of the normal indicatrix of a regular curve $\alpha$ and its Mannheim-direction curves. 
Proposition 16. Let $\alpha$ be a curve in $E^{3}, \alpha_{N}$ be normal indicatrix of a curve $\alpha$ and $\beta$ is Mannheim-direction curve of $\alpha_{N}$. The relationships between curvatures of the curves $\alpha_{N}$ and $\beta$ can be expressed as follows:

and

$$
k_{\beta}=\tau_{N} \cos \left(\int k_{N}\left(s_{N}\right) d s\right) \quad, \quad \tau_{\beta}=\tau_{N} \sin \left(\int k_{N}\left(s_{N}\right) d s_{N}\right)
$$

$$
k_{N}=\frac{k_{\beta}^{2}}{k_{\beta}^{2}+\tau_{\beta}^{2}}\left(\frac{\tau_{\beta}}{k_{\beta}}\right)^{\prime}, \quad \tau_{N}=\sqrt{k_{\beta}^{2}+\tau_{\beta}^{2}} .
$$

Proof. From (11) we have:

$$
k_{\beta}=\tau_{N} \cos \left(\int k_{N}\left(s_{N}\right) d s_{N}\right) .
$$

Since $\beta$ is a Mannheim-direction curve of $\alpha_{N}$, then the Frenet frame of a curve $\beta$ can be expressed by:

$$
\left\{\begin{array}{l}
T_{\beta}=\sin \left(\int k_{N}\left(s_{N}\right) d s_{N}\right) T_{N}+\cos \left(\int k_{N}\left(s_{N}\right) d s_{N}\right) N_{N} \\
N_{\beta}=B_{N} \\
B_{\beta}=\cos \left(\int k_{N}\left(s_{N}\right) d s_{N}\right) T_{N}-\sin \left(\int k_{N}\left(s_{N}\right) d s_{N}\right) N_{N} .
\end{array}\right.
$$

By the definition, we obtain the torsion as following

$$
\tau_{\beta}=\tau_{N} \sin \left(\int k_{N}\left(s_{N}\right) d s_{N}\right) .
$$

From (13) and (14) we have

$$
\tau_{N}=\sqrt{k_{\beta}^{2}+\tau_{\beta}^{2}},
$$

and

By differentiating the last equality, we obtain

$$
\int k_{N}\left(s_{N}\right) d s_{N}=\arctan \left(\frac{\tau_{\beta}}{k_{\beta}}\right) .
$$

$$
k_{N}=\frac{k_{\beta}^{2}}{k_{\beta}^{2}+\tau_{\beta}^{2}}\left(\frac{\tau_{\beta}}{k_{\beta}}\right)^{\prime}
$$

Corollary 17. Let $\beta$ be Mannheim-direction curve of $\alpha_{N}$, the normal indicatrix of a curve $\alpha$, then:

$$
\begin{aligned}
\frac{\sigma^{\prime}}{f\left(1+\sigma^{2}\right)} & =\tau_{N}=\frac{\sqrt{k_{\beta}^{2}+\tau_{\beta}^{2}}}{\Gamma}=\frac{\tau_{N}}{k_{N}}=\frac{1}{\frac{k_{\beta}^{2}}{\left(k_{\beta}^{2}+\tau_{\beta}^{2}\right)^{\frac{3}{2}}}\left(\frac{\tau_{\beta}}{k_{\beta}}\right)^{\prime}} .
\end{aligned}
$$

By using (1) and from Corollary (17), we can give the two following theorems without any proof.

Theorem 18. Let $\alpha$ be a curve in $E^{3}, \alpha_{N}$ be normal indicatrix of a curve $\alpha$ and $\beta$ be Mannheim-direction curve of $\alpha_{N}$. Then the followings are equivalent:

i- $\alpha$ is a slant helix.

ii- $\alpha_{N}$ is a circle or a part of a circle on the unit sphere.

iii- $\beta$ is a straight line.

Theorem 19. Let $\alpha$ be a curve in $E^{3}, \alpha_{N}$ be normal indicatrix of a curve $\alpha$ and $\beta$ be Mannheim-direction curve of $\alpha_{N}$. Then the followings are equivalent:

i- $\alpha$ is a C-slant helix.

ii- $\alpha_{N}$ is a spherical helix.

iii- $\beta$ is a slant helix. 


\section{Examples}

Example 20. Consider a unit-speed slant helix

$$
\alpha(s)=\left(\frac{2}{5} \sin (2 s)-\frac{1}{40} \sin (8 s) ;-\frac{2}{5} \cos (2 s)+\frac{1}{40} \cos (8 s) ; \frac{4}{15} \sin (3 s)\right)
$$

Let $N(s)$ be the principal vector field of the given curve $\alpha$,

$$
N(s)=\left(\frac{4}{5} \cos (5 s) ; \frac{4}{5} \sin (5 s) ; \frac{-3}{5}\right) .
$$

Since $s_{N}=4 s$, then the normal indicatrix $\alpha_{N}$ of the curve $\alpha$ is obtained as follows:

$$
\alpha_{N}\left(s_{N}\right)=\left(\frac{4}{5} \cos \left(\frac{5}{4} s_{N}\right) ; \frac{4}{5} \sin \left(\frac{5}{4} s_{N}\right) ; \frac{-3}{5}\right)
$$

The Frenet-Serret apparatus of $\alpha_{N}$ is given as following:

$$
\left\{\begin{aligned}
T_{N}\left(s_{N}\right) & =\left(-\sin \left(\frac{5}{4} s_{N}\right) ; \cos \left(\frac{5}{4} s_{N}\right) ; 0\right) \\
N_{N}\left(s_{N}\right) & =\left(-\cos \left(\frac{5}{4} s_{N}\right) ;-\sin \left(\frac{5}{4} s_{N}\right) ; 0\right) \\
B_{N}\left(s_{N}\right) & =(0 ; 0 ; 1) \\
k_{N}\left(s_{N}\right) & =\frac{5}{4} \\
\tau_{N}\left(s_{N}\right) & =0
\end{aligned}\right.
$$

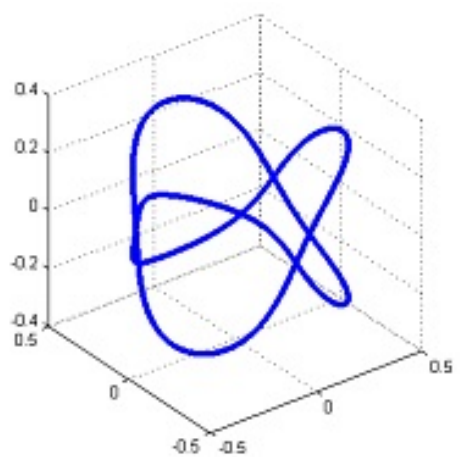

Figure 1. Slant helix $\alpha$

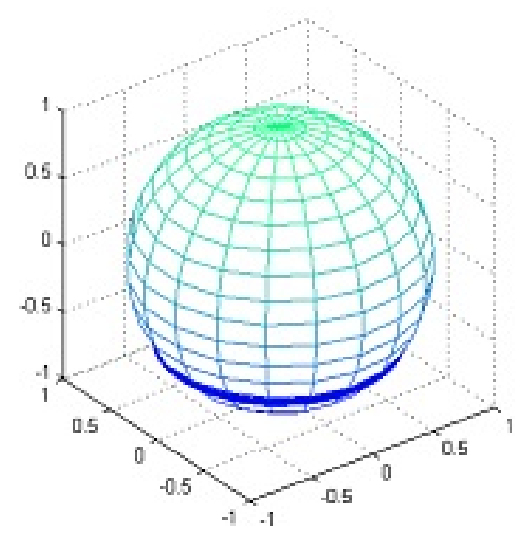

Figure 2. Normal indicatrix $\alpha \_\mathrm{N}$ of the slant helix $\alpha$

Lets $\beta_{E}$ and $\beta_{M}$ be evolute-direction curve and Mannheim-direction curve of the normal indicatrix $\alpha_{N}$ of the curve $\alpha$, respectively. Then $\beta_{E}$ and $\beta_{M}$, are respectively, the integral curves of the vector fields $X_{E}$ and $X_{M}$, where

$$
\left\{\begin{array}{l}
X_{E}\left(s_{N}\right)=\left(-\cos \left(\frac{5}{4} s_{N}\right) \sin \left(\theta_{1}\right) ;-\sin \left(\frac{5}{4} s_{N}\right) \sin \left(\theta_{1}\right) ; \cos \left(\theta_{1}\right)\right), \\
X_{M}\left(s_{N}\right)=\left(-\cos \left(\theta_{2}\right) ; \sin \left(\theta_{2}\right) ; 0\right)
\end{array}\right.
$$

Therefore $\beta_{E}$ and $\beta_{M}$ are respectively obtained as following

$$
\left\{\begin{array}{l}
\beta_{E}\left(s_{\beta}\right)=\left(-\frac{4}{5} \sin \left(\frac{5}{4} s_{\beta}\right) \sin \left(\theta_{1}\right)+c_{1} ; \frac{4}{5} \cos \left(\frac{5}{4} s_{\beta}\right) \sin \left(\theta_{1}\right)+c_{2} ; \cos \left(\theta_{1}\right) s_{\beta}+c_{3}\right), \\
\beta_{M}\left(s_{\beta}\right)=\left(-\cos \left(\theta_{2}\right) s_{\beta}+c_{4} ; \sin \left(\theta_{2}\right) s_{\beta}+c_{5} ; c_{6}\right)
\end{array}\right.
$$


where $\theta_{1}, \theta_{2}$ and $c_{i},\{i=1, . ., 6\}$ are reals constants. By giving $\theta_{1}=\theta_{2}=\frac{\pi}{4}$ and $c_{i}=0$, we obtain the curves $\beta_{E}$ and $\beta_{M}$ as illustrate in the following figures

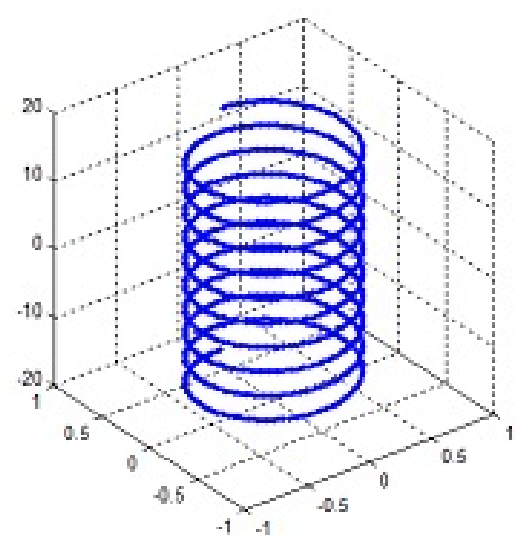

Figure 3. Evolute-direction curve of the normal indicatrix $\alpha_{N}$ of $\alpha$

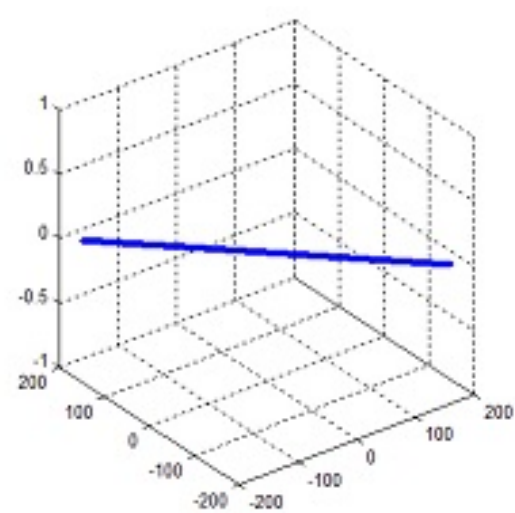

Figure 4. Mannheim-direction curve of the normal indicatrix $\alpha_{N}$ of $\alpha$

Example 21. In this example, we construct approximately a $C$-slant helix with using Taylor expansion .

In (D.J.Struik (1961)), author has given the following form of a curve using the Frenet vectors of the curve and Taylor expansion

$$
\alpha(s)=\alpha(0)+\left(s-\frac{s^{3} k^{2}}{6}\right) T+\left(\frac{s^{2} k}{2}-\frac{s^{3} k^{\prime}}{6}\right) N+\frac{s^{3} k \tau}{6} B+o\left(s^{3}\right) .
$$

Let $k$ and $\tau$ be the curvature and the torsion of the C-slant helix $\alpha$ given in C. Ramis. B. Uzunoglu \& Y. Yayli (2014) as following

$$
\begin{aligned}
& k(s)=\sin (s) \cos (\sin (s)), \\
& \tau(s)=\sin (s) \sin (\sin (s)),
\end{aligned}
$$

then we obtain the curve $\alpha$ as illustrate in the figure (5).

Let $N(s)$ be the principal vector field of the given curve $\alpha$,

$$
\begin{aligned}
N(s)= & \left(\frac{\sqrt{2}+1}{2 \sqrt{2}} \cos ((\sqrt{2}-1) s)-\frac{\sqrt{2}-1}{2 \sqrt{2}} \cos ((\sqrt{2}+1) s),\right. \\
& \frac{\sqrt{2}+1}{2 \sqrt{2}} \sin ((\sqrt{2}-1) s)-\frac{\sqrt{2}-1}{2 \sqrt{2}} \sin ((\sqrt{2}+1) s), \\
& \left.\frac{1}{\sqrt{2}} \cos s\right),
\end{aligned}
$$

since the arc-length parameter of $N(s)$ is $s_{N}=-\cos (s)$, the normal indicatrix $\alpha_{N}$ of the given curve $\alpha$, is obtained as follows:

$$
\begin{aligned}
\alpha_{N}\left(s_{N}\right)= & \left(\frac{\sqrt{2}+1}{2 \sqrt{2}} \cos \left((\sqrt{2}-1) \arccos \left(-s_{N}\right)\right)-\frac{\sqrt{2}-1}{2 \sqrt{2}} \cos \left((\sqrt{2}+1) \arccos \left(-s_{N}\right)\right),\right. \\
& \frac{\sqrt{2}+1}{2 \sqrt{2}} \sin \left((\sqrt{2}-1) \arccos \left(-s_{N}\right)\right)-\frac{\sqrt{2}-1}{2 \sqrt{2}} \sin \left((\sqrt{2}+1) \arccos \left(-s_{N}\right)\right), \\
& \left.\frac{-s_{N}}{\sqrt{2}}\right) .
\end{aligned}
$$


The Frenet-Serret apparatus of $\alpha_{N}$ is given as following:

$$
\begin{aligned}
& T_{N}\left(s_{N}\right)=\left(\frac{-1}{2 \sqrt{2} \sqrt{1-s_{N}^{2}}} \sin \left((\sqrt{2}-1) \arccos \left(-s_{N}\right)\right)+\frac{1}{2 \sqrt{2} \sqrt{1-s_{N}^{2}}} \sin \left((\sqrt{2}+1) \arccos \left(-s_{N}\right)\right),\right. \\
& \frac{1}{2 \sqrt{2} \sqrt{1-s_{N}^{2}}} \cos \left((\sqrt{2}-1) \arccos \left(-s_{N}\right)\right)-\frac{1}{2 \sqrt{2} \sqrt{1-s_{N}^{2}}} \cos \left((\sqrt{2}+1) \arccos \left(-s_{N}\right)\right) \\
& \left.\frac{-1}{\sqrt{2}}\right) \\
& N_{N}\left(s_{N}\right)=\left(\frac{s_{N}}{2 \sqrt{2}\left(1-s_{N}^{2}\right)}\left[\sin \left((\sqrt{2}+1) \arccos \left(-s_{N}\right)\right)-\sin \left((\sqrt{2}-1) \arccos \left(-s_{N}\right)\right)\right]\right. \\
& +\frac{1}{\sqrt{1-s_{N}^{2}}}\left[-\frac{\sqrt{2}-1}{2 \sqrt{2}} \cos \left((\sqrt{2}-1) \arccos \left(-s_{N}\right)\right)+\frac{\sqrt{2}+1}{2 \sqrt{2}} \cos \left((\sqrt{2}+1) \arccos \left(-s_{N}\right)\right)\right], \\
& -\frac{s_{N}}{2 \sqrt{2}\left(1-s_{N}^{2}\right)}\left[\cos \left((\sqrt{2}+1) \arccos \left(-s_{N}\right)\right)-\cos \left((\sqrt{2}-1) \arccos \left(-s_{N}\right)\right)\right] \\
& +\frac{1}{\sqrt{1-s_{N}^{2}}}\left[-\frac{\sqrt{2}-1}{2 \sqrt{2}} \sin \left((\sqrt{2}-1) \arccos \left(-s_{N}\right)\right)+\frac{\sqrt{2}+1}{2 \sqrt{2}} \sin \left((\sqrt{2}+1) \arccos \left(-s_{N}\right)\right)\right], \\
& 0) \text {, } \\
& \begin{aligned}
B_{N}\left(s_{N}\right)= & \left(-\frac{s_{N}}{4\left(1-s_{N}^{2}\right)}\left[\cos \left((\sqrt{2}+1) \arccos \left(-s_{N}\right)\right)-\cos \left((\sqrt{2}-1) \arccos \left(-s_{N}\right)\right)\right]\right. \\
& +\frac{1}{\sqrt{2} \sqrt{1-s_{N}^{2}}}\left[-\frac{\sqrt{2}-1}{2 \sqrt{2}} \sin \left((\sqrt{2}-1) \arccos \left(-s_{N}\right)\right)+\frac{\sqrt{2}+1}{2 \sqrt{2}} \sin \left((\sqrt{2}+1) \arccos \left(-s_{N}\right)\right)\right], \\
& -\frac{s_{N}}{4\left(1-s_{N}^{2}\right)}\left[\sin \left((\sqrt{2}+1) \arccos \left(-s_{N}\right)\right)-\sin \left((\sqrt{2}-1) \arccos \left(-s_{N}\right)\right)\right] \\
& -\frac{1}{\sqrt{2} \sqrt{1-s_{N}^{2}}}\left[-\frac{\sqrt{2}-1}{2 \sqrt{2}} \cos \left((\sqrt{2}-1) \arccos \left(-s_{N}\right)\right)+\frac{\sqrt{2}+1}{2 \sqrt{2}} \cos \left((\sqrt{2}+1) \arccos \left(-s_{N}\right)\right)\right], \\
& \left.\frac{1}{\sqrt{2}}\right),
\end{aligned} \\
& k_{N}\left(s_{N}\right)=\frac{1}{\sqrt{1-s_{N}^{2}}} \\
& \tau_{N}\left(s_{N}\right)=-\frac{1}{\sqrt{1-s_{N}^{2}}} .
\end{aligned}
$$




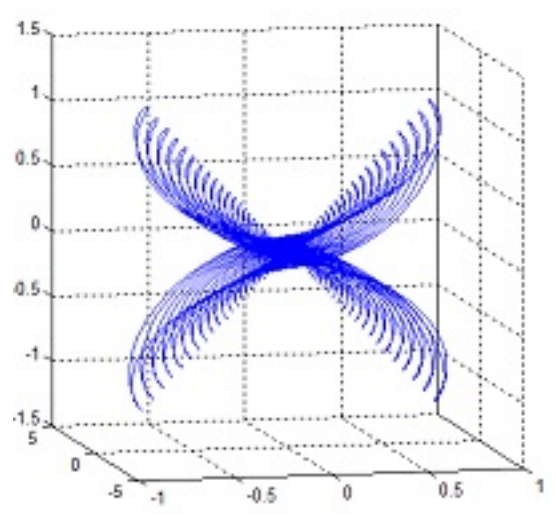

Figure 5. C-slant helix $\alpha$

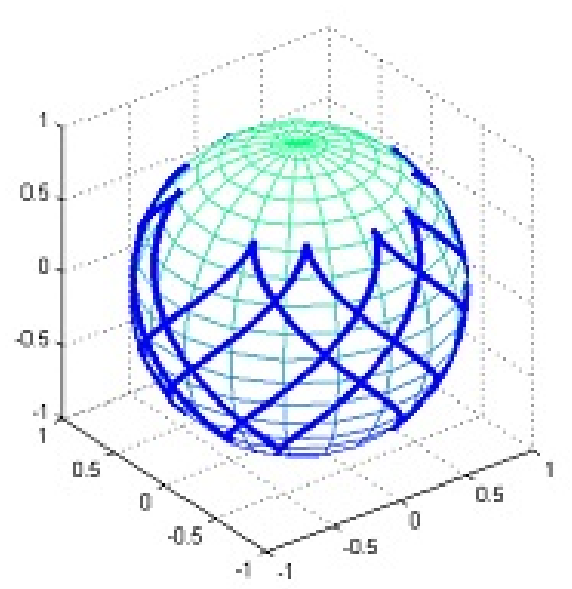

Figure 6. Normal indicatrix $\alpha_{N}$ of the C-slant helix $\alpha$

Lets $\beta_{E}, \beta_{B}$ and $\beta_{M}$, be evolute-direction curve, Bertrand-direction curve and Mannheim-direction curve of the normal indicatrix $\alpha_{N}$ of the curve $\alpha$, respectively. Then $\beta_{E}, \beta_{B}$ and $\beta_{M}$, are respectively, the integral curves of the vector fields $X_{E}$, $X_{B}$ and $X_{M}$, where

$$
\begin{aligned}
X_{E}\left(s_{N}\right)= & \left(-\frac{\sqrt{2}-1}{2 \sqrt{2}} \sin \left((\sqrt{2}+1) \arccos \left(-s_{N}\right)-\theta_{3}\right)-\frac{\sqrt{2}+1}{2 \sqrt{2}} \sin \left((\sqrt{2}-1) \arccos \left(-s_{N}\right)+\theta_{3}\right),\right. \\
& \frac{\sqrt{2}+1}{2 \sqrt{2}} \cos \left((\sqrt{2}-1) \arccos \left(-s_{N}\right)+\theta_{3}\right)+\frac{\sqrt{2}-1}{2 \sqrt{2}} \cos \left((\sqrt{2}+1) \arccos \left(-s_{N}\right)-\theta_{3}\right), \\
& \left.\frac{1}{\sqrt{2}} s_{N} \sin \left(\theta_{3}\right)+\frac{1}{\sqrt{2}} \sqrt{1-s_{N}^{2}} \cos \left(\theta_{3}\right)\right), \\
X_{B}\left(s_{N}\right)= & \left(\frac{1}{\sqrt{2}} \cos \left(\sqrt{2} \arccos \left(-s_{N}\right)\right)(\cos \theta+\sin \theta),\right. \\
& \frac{1}{\sqrt{2}} \sin \left(\sqrt{2} \arccos \left(-s_{N}\right)\right)(\cos \theta+\sin \theta), \\
& \left.-\frac{1}{\sqrt{2}}(\cos \theta-\sin \theta)\right) . \\
& \left.-\frac{1}{\sqrt{2}} s_{N} \cos \left(\theta_{4}\right)-\frac{1}{\sqrt{2}} \sqrt{1-s_{N}^{2}} \sin \left(\theta_{4}\right)\right) . \\
& -\frac{\sqrt{2}+1}{2 \sqrt{2}} \sin \left((\sqrt{2}-1) \arccos \left(-s_{N}\right)-\theta_{4}\right)+\frac{\sqrt{2}-1}{2 \sqrt{2}} \sin \left((\sqrt{2}+1) \arccos \left(-s_{N}\right)+\theta_{4}\right), \\
X_{M}\left(s_{N}\right)= & \left(\frac{\sqrt{2}-1}{2 \sqrt{2}} \cos \left((\sqrt{2}+1) \arccos \left(-s_{N}\right)+\theta_{4}\right)-\frac{\sqrt{2}+1}{2 \sqrt{2}} \cos \left((\sqrt{2}-1) \arccos \left(-s_{N}\right)-\theta_{4}\right),\right. \\
& \\
& \\
& \\
&
\end{aligned}
$$


Therefore $\beta_{E}, \beta_{B}$ and $\beta_{M}$ are respectively obtained as following:

$$
\begin{aligned}
& \beta_{E}(s)=\left(\frac { 1 } { 8 } \left[(-1+\sqrt{2}) \sin \left(\theta_{3}-\sqrt{2} s\right)+(1+\sqrt{2}) \sin \left(\theta_{3}+\sqrt{2} s\right)+(3+2 \sqrt{2}) \sin \left(\theta_{3}+(-2+\sqrt{2}) s\right)\right.\right. \\
& \left.+(-3+2 \sqrt{2}) \sin \left(\theta_{3}-(2+\sqrt{2}) s\right)\right]+c_{7}, \\
& \frac{1}{8}\left[(-1+\sqrt{2}) \cos \left(\theta_{3}-\sqrt{2} s\right)-(1+\sqrt{2}) \cos \left(\theta_{3}+\sqrt{2} s\right)-(3+2 \sqrt{2}) \cos \left(\theta_{3}+(-2+\sqrt{2}) s\right)\right. \\
& \left.+(-3+2 \sqrt{2}) \cos \left(\theta_{3}-(2+\sqrt{2}) s\right)\right]+c_{8} \\
& \left.\frac{1}{4 \sqrt{2}}\left(2 s \cos \left(\theta_{3}\right)+\sin \left(\theta_{3}-2 s\right)\right)+c_{9}\right), \\
& \beta_{B}(s)=\left(\frac{1}{2}(2 \sin (s) \sin (\sqrt{2} s)+\sqrt{2} \cos (s) \cos (\sqrt{2} s))(\cos \theta+\sin \theta)+c_{10},\right. \\
& \frac{1}{2}(\sqrt{2} \cos (s) \sin (\sqrt{2} s)-2 \sin (s) \cos (\sqrt{2} s))(\cos \theta+\sin \theta)+c_{11}, \\
& \left.\frac{1}{\sqrt{2}} \cos (s)(\cos \theta+\sin \theta)+c_{12}\right), \\
& \beta_{M}(s)=\left(\frac { 1 } { 8 } \left[(1+\sqrt{2}) \cos \left(\theta_{4}-\sqrt{2} s\right)+(-1+\sqrt{2}) \cos \left(\theta_{4}+\sqrt{2} s\right)+(3+2 \sqrt{2}) \cos \left(\theta_{4}+(2-\sqrt{2}) s\right)\right.\right. \\
& \left.+(-3+2 \sqrt{2}) \cos \left(\theta_{4}+(2+\sqrt{2}) s\right)\right]+c_{13}, \\
& \frac{1}{8}\left[-(1+\sqrt{2}) \sin \left(\theta_{4}-\sqrt{2} s\right)+(-1+\sqrt{2}) \sin \left(\theta_{4}+\sqrt{2} s\right)-(3+2 \sqrt{2}) \sin \left(\theta_{4}+(2-\sqrt{2}) s\right)\right. \\
& \left.+(-3+2 \sqrt{2}) \sin \left(\theta_{4}+(2+\sqrt{2}) s\right)\right]+c_{14}, \\
& \left.-\frac{1}{4 \sqrt{2}}\left(2 s \sin \left(\theta_{4}\right)+\cos \left(\theta_{4}+2 s\right)\right)+c_{15}\right),
\end{aligned}
$$

where $c_{j},\{j=7, . ., 15\}$ are reals constants. By giving $c_{j}=0, \theta_{3}=\theta=\frac{\pi}{3}$ and $\theta_{4}=0$, we obtain the curves $\beta_{E}, \beta_{B}$ and $\beta_{M}$ as illustrate in the followings figures: 


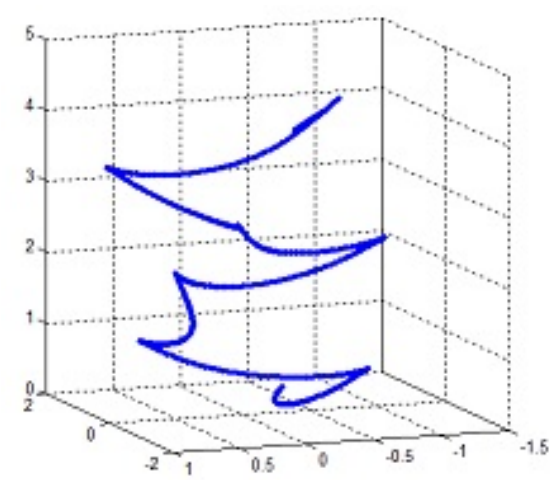

Figure 7. Evolute-direction curve of the normal indicatrix $\alpha_{N}$ of $\alpha$

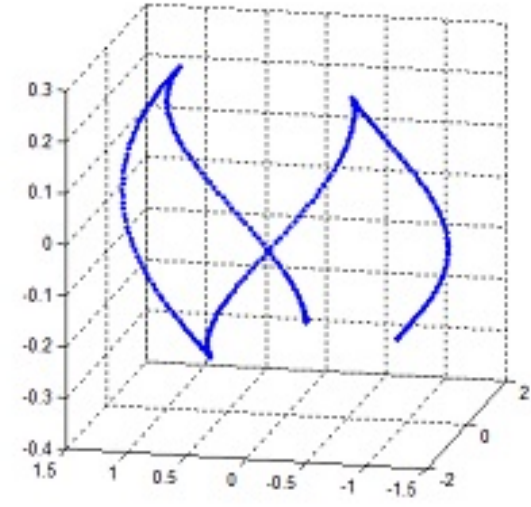

Figure 8. Bertrand-direction curve of the normal indicatrix $\alpha_{N}$ of $\alpha$

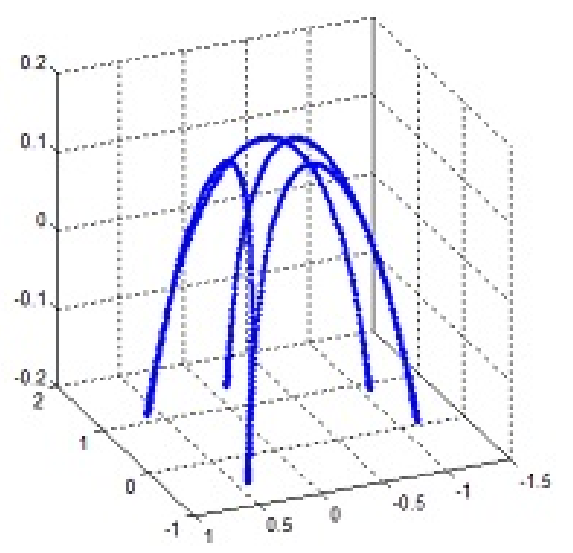

Figure 9. Mannheim-direction curve of the normal indicatrix $\alpha_{N}$ of $\alpha$

\section{References}

Ali, A. T. (2009). New special curves and their spherical indicatrices. arXiv preprint arXiv: 0909.2390.

Beyhan, U., Gök, İ., \& Yayli, Y. (2016). A new approach on curves of constant precession. Applied Mathematics and Computation, 275, 317-323.

Choi, J. H., \& Kim, Y. H. (2012). Associated curves of a Frenet curve and their applications. Applied Mathematics and Computation, 218(18), 9116-9124.

Choi, J. H., Kim, Y. H., \& Ali, A. T. (2012). Some associated curves of Frenet non-lightlike curves in $E_{1}^{3}$. J. Math. Anal. Appl., 394, 712-723.

Kőrpinar, T., Sariaydin, M. T., \& Turhan, E. (2013). Associated curves according to Bishop frame in Euclidean 3-space. Advanced Modeling and Optimization, 15(3), 713-717.

Macit, N., \& Düldül, M. (2014). Some new associated curves of a Frenet curve in $E^{3}$ and $E^{4}$. Turk. J. Math., 38(6), 1023-1037.

Önnder, M. (2017). Construction of curve pairs and their applications. arXiv preprint arXiv: 1701.04812.

Qian, J., \& Kim, Y. H. (2015). Directional associated curves of a null curve in Minkowski 3-space. Bull. Korean Mathematical Society, 52(1), 183-200.

Ramis, C., Uzunoglu, B., \& Yayli, Y. (2014). New associated curves k-principale direction curves and $N_{k}$ slant helix. arXiv preprint arXiv: 1404.7369 .

Şahiner, B. (2019). Direction curves of tangent indicatrix of a curve. Applied Mathematics and Computation, 343, 
273-284.

Struik, D. J. (1961). Lectures on classical differential geometry. courier corporation.

\section{Copyrights}

Copyright for this article is retained by the author(s), with first publication rights granted to the journal.

This is an open-access article distributed under the terms and conditions of the Creative Commons Attribution license (http://creativecommons.org/licenses/by/4.0/). 